\title{
Berry curvature engineering by gating two-dimensional antiferromagnets
}

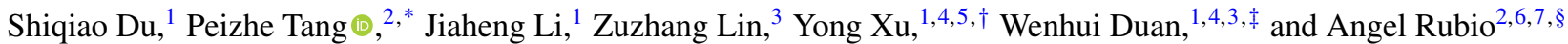 \\ ${ }^{1}$ State Key Laboratory of Low-Dimensional Quantum Physics, Department of Physics, Tsinghua University, Beijing 100084, China \\ ${ }^{2}$ Max Planck Institute for the Structure and Dynamics of Matter, Center for Free-Electron Laser Science, \\ Luruper Chaussee 149, 22761 Hamburg, Germany \\ ${ }^{3}$ Institute for Advanced Study, Tsinghua University, Beijing 100084, China \\ ${ }^{4}$ Collaborative Innovation Center of Quantum Matter, Beijing 100084, China \\ ${ }^{5}$ RIKEN Center for Emergent Matter Science (CEMS), Wako, Saitama 351-0198, Japan \\ ${ }^{6}$ Nano-Bio Spectroscopy Group and ETSF, Dpto. Fisica de Materiales, Universidad del País Vasco UPV/EHU, 20018 San Sebastián, Spain \\ ${ }^{7}$ Center for Computational Quantum Physics, Flatiron Institute, 162 Fifth Avenue, New York, New York 10010, USA
}

(Received 4 October 2019; accepted 8 April 2020; published 4 May 2020)

\begin{abstract}
Recent advances in tuning electronic, magnetic, and topological properties of two-dimensional (2D) magnets have opened a new frontier in the study of quantum physics and promised exciting possibilities for future quantum technologies. In this study, we find that the dual-gate technology can well tune the electronic and topological properties of antiferromagnetic (AFM) even septuple-layer (SL) $\mathrm{MnBi}_{2} \mathrm{Te}_{4}$ thin films. Under an out-of-plane electric field that breaks $\mathcal{P} \mathcal{T}$ symmetry, the Berry curvature of the thin film could be engineered efficiently, resulting in a huge change of anomalous Hall (AH) signal. Beyond the critical electric field, the double-SL $\mathrm{MnBi}_{2} \mathrm{Te}_{4}$ thin film becomes a Chern insulator with a high Chern number of 3 . We further demonstrate that such 2D material can be used as an AFM switch via electric-field control of the AH signal. These discoveries inspire the design of low-power memory prototypes for future AFM spintronic applications.
\end{abstract}

DOI: 10.1103/PhysRevResearch.2.022025

Antiferromagnetic spintronics (AFMS), aiming to use antiferromagnets to complement or replace ferromagnets as active components of spintronic devices, opens a new era in the field of spintronics owing to its numerous advantages, including robustness against perturbation of external magnetic fields, the absence of a stray field, and ultrafast dynamics [1-5]. The key issue to design AFMS devices is to find an efficient approach to manipulate and detect the magnetic or electronic quantum states of an antiferromagnet. By using spin-transfer torque and spin-orbit torque [1,5], external electric currents are able to write information by controlling the AFM order inside spintronic devices [6-10] or by generating spin currents at interfaces between antiferromagnets and nonmagnets [11]. For example, via applying local current in the tetragonal CuMnAs thin film, AFM spin orientations can be manipulated for information storage, which can be a readout via detecting the anisotropic magnetoresistance [12,13]. Based on such a proposal, room-temperature AFM memory cells have been fabricated experimentally [14].

\footnotetext{
*peizhe.tang@mpsd.mpg.de

†yongxu@mail.tsinghua.edu.cn

$\ddagger$ dwh@phys.tsinghua.edu.cn

§angel.rubio@mpsd.mpg.de
}

Published by the American Physical Society under the terms of the Creative Commons Attribution 4.0 International license. Further distribution of this work must maintain attribution to the author(s) and the published article's title, journal citation, and DOI. Open access publication funded by the Max Planck Society.
Electric detection of the anomalous Hall (AH) effect provides an alternative powerful scenario to design spintronic devices based on antiferromagnets. The physical origin of the AH effect has been under debate for decades [15]. In recent years, people have realized that Berry curvature $\boldsymbol{\Omega}(\mathbf{k})$ in the momentum space plays an important role in generating the intrinsic AH effect [15-17]. Based on the symmetry argument, the Berry curvature could be nonzero in a system without the combination of time-reversal symmetry $(\mathcal{T})$ and inversion symmetry $(\mathcal{P})$, namely, $\mathcal{P} \mathcal{T}$ symmetry. Such a constraint indicates antiferromagnets with noncollinear magnetism and antiferromagnet heterostructures as potential material candidates to possess the nonzero Berry curvature, including $\mathrm{MnPSe}_{3}$ [18], $\mathrm{Mn}_{3} \mathrm{Ge}$ [19-21], $\mathrm{Mn}_{3} \mathrm{Sn}$ [22], $\mathrm{Mn}_{3} \mathrm{Ir}$ [23], $\mathrm{Mn}_{3} \mathrm{Pt} / \mathrm{BaTiO}_{3}$ [24], and $\mathrm{CrSb} / \mathrm{Cr}$-doped $(\mathrm{Bi}, \mathrm{Sb})_{2} \mathrm{Te}_{3}$ superlattices [25]. However, the external control of their $\mathrm{AH}$ signal is challenging in practice. For AFM structures with collinear magnetisms, the possible existence of $\mathcal{P} \mathcal{T}$ symmetry guarantees the vanishing of the Berry curvature. But some three-dimensional (3D) antiferromagnets still contain the Dirac and coupled Weyl fermions in their 3D bulk states [26], whose topological properties can be manipulated via spin-orbit torque [27]. These phenomena lend us a fresh perspective regarding how to manipulate and engineer the Berry curvature in AFM materials with collinear magnetisms, which is essential to generate $\mathrm{AH}$ signals as the detection in spintronic devices.

The recently discovered 3D AFM topological insulator (TI) $\mathrm{MnBi}_{2} \mathrm{Te}_{4}$ provides us a chance to design topological quantum devices controlled by external fields. Similar to the prototype of 3D TI (e.g., the $\mathrm{Bi}_{2} \mathrm{Se}_{3}$ family), the $\mathrm{MnBi}_{2} \mathrm{Te}_{4}$ 
crystal is a layered material [28-31]. There exists strong chemical bonding within each septuple layer (SL) (consisting of Te-Bi-Te-Mn-Te-Bi-Te) and weak van der Waals bonding between SLs. Each Mn atom has a magnetic moment of $\sim 5 \mu_{\mathbf{B}}$ ( $\mu_{\mathbf{B}}$ is the Bohr magneton), and its spin orientation is along the out-of-plane $z$ direction [31,32]. In the ground state, a long-range ferromagnetic order is formed in each SL, and adjacent SLs couple with each other antiferromagnetically, displaying a Néel temperature $T_{N} \sim 25 \mathrm{~K}[33,34]$. In contrast to AFM transition-metal oxides [35], ultrathin films of layered $\mathrm{MnBi}_{2} \mathrm{Te}_{4}$ can be easily cleaved by mechanical exfoliation [36-38] or grown via molecular beam epitaxy [28]. The quantized Hall effect without Landau levels has been theoretically predicted and experimentally observed in these thin films [31,32,36-38].

Herein, we focus on a $\mathrm{MnBi}_{2} \mathrm{Te}_{4}$ thin film with double SL whose intrinsic electronic property is topologically trivial. By using $a b$ initio calculations, we demonstrate that the out-ofplane electric field induced by dual-gate technology breaks $\mathcal{P} \mathcal{T}$ symmetry in AFM double-SL $\mathrm{MnBi}_{2} \mathrm{Te}_{4}$ thin film, engineers its band structures significantly, and generates huge $\mathrm{AH}$ signals. Beyond the critical value, the electric field induces a topological phase transition, driving the double-SL $\mathrm{MnBi}_{2} \mathrm{Te}_{4}$ to be a quantized anomalous Hall (QAH) insulator with a high Chern number of 3. Based on these useful properties, we propose a prototype device as the electric-field-controlled topological AFM memory, whose performance is expected to be much higher than the current AFM random-access memory. Thanks to the AFM ground state, its electric switch should be stable and robust under an external magnetic field, and the switching time is very fast.

For the insulating AFM bilayer thin film with $\mathcal{P} \mathcal{T}$ symmetry, such as double-SL $\mathrm{MnBi}_{2} \mathrm{Te}_{4}$, every Bloch state at any $\mathbf{k}$ point is doubly degenerated. Figure 1 demonstrates the schematics of AFM thin-film structures in real space, with and without an out-of-plane electric field, and corresponding electronic bands in momentum space. In the intrinsic film [see Figs. 1(a) and 1(c)], the Berry curvature of the $n$th Bloch band satisfies $\boldsymbol{\Omega}_{n}(\mathbf{k})=-\boldsymbol{\Omega}_{n}(-\mathbf{k})$ with $\mathcal{T}$ symmetry, and $\mathcal{P}$ symmetry enforces $\boldsymbol{\Omega}_{n}(\mathbf{k})=\boldsymbol{\Omega}_{n}(-\mathbf{k})$. Thus, $\boldsymbol{\Omega}_{n}(\mathbf{k})$ is zero in the presence of $\mathcal{P} \mathcal{T}$ symmetry. Due to the two-dimensional (2D) geometric property, the double-SL $\mathrm{MnBi}_{2} \mathrm{Te}_{4}$ can be applied with an out-of-plane electric field easily by using the dual-gate technology. As shown in Fig. 1(b), the electrostatic potential is different in each $\mathrm{SL}$, which breaks the $\mathcal{P} \mathcal{T}$ symmetry. The double degeneracy of each band gets broken and $\boldsymbol{\Omega}_{n}(\mathbf{k})$ becomes nonzero, which enables the possible observation of an AH effect via changing the carrier density in this system. In Figs. 1(d) and 1(e), we show the schematics of the change of electronic structures under the electric field. On the edge of the top valance band, the degenerated states are mainly contributed by out-of-plane orbitals with opposite spins localized at different SLs. The spatial variance of the electrostatic potential can split the band edge, giving rise to a Zeeman-like band splitting. On the other hand, in-plane orbitals are major components on the edge of the bottom conduction band, and the applied electric field results in a Rashba-like spin splitting. When increasing the magnitude of the electric field, the band gap decreases gradually. At the critical value, the band gap closes, which possibly leads to a topological phase transition. (a)

(b)

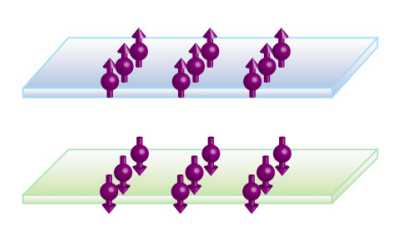

$p \cdot \mathcal{T}, \Omega(\mathrm{k})=0$

(c)

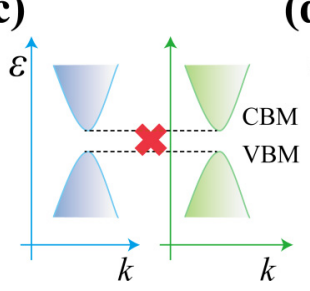

(d)

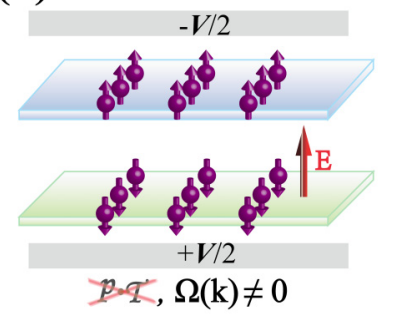

(e)

FIG. 1. (a) Schematic of AFM double layer with $\mathcal{P} \mathcal{T}$ symmetry. The purple balls in each layer represent magnetic atoms whose magnetic orientations (out of plane) are depicted by arrows (e.g., $\mathrm{Mn}$ atoms in double-SL $\mathrm{MnBi}_{2} \mathrm{Te}_{4}$ ). (b) Schematic of AFM double layer with an out-of-plane electric field in which $\mathcal{P} \mathcal{T}$ symmetry is broken. (c)-(e) Schematics of band structures for AFM double-SL $\mathrm{MnBi}_{2} \mathrm{Te}_{4}$ thin film without and with electric field. The colors of blue and green stand for states with different spin. In (c), each band is doubly degenerate. In (d) and (e), under the electric field, $\mathcal{P} \mathcal{T}$ symmetry is broken. The dot lines in (e) stand for the original bands without electric field.

By using density functional theory, we calculate electronic structures of the double-SL $\mathrm{MnBi}_{2} \mathrm{Te}_{4}$ thin film with and without an electric field to verify the above physical picture. Figure 2(a) displays the lattice structure of double-SL $\mathrm{MnBi}_{2} \mathrm{Te}_{4}$ and its first Brillouin zone (BZ). The calculated band structures and Berry curvature $\boldsymbol{\Omega}(\mathbf{k})$ for occupied bands are shown in Figs. 2(b)-2(k). In the absence of external field, the AFM ground state has neither $\mathcal{P}$ symmetry nor $\mathcal{T}$ symmetry, but has the $\mathcal{P} \mathcal{T}$ symmetry and $\mathcal{C}_{3 z}$ rotational symmetry along the $z$ direction. The intrinsic double-SL $\mathrm{MnBi}_{2} \mathrm{Te}_{4}$ is a trivial semiconductor with a direct band gap at the $\Gamma$ point, whose gap size is $76 \mathrm{meV}$ from the Perdew-Burke-Ernzerhof (PBE) functional and $\boldsymbol{\Omega}(\mathbf{k})$ is zero. We find that each SL in $\mathrm{MnBi}_{2} \mathrm{Te}_{4}$ thin film is antiferromagnetically coupled with the other in the ground state, consistent with previous calculations [31,32].

When applying an out-of-plane electric field to the thin film, its magnetism keeps the AFM order as the ground state in a finite field range. The effect of the electric field on the lattice is also negligible (for details, see Supplemental Material [39]). But the electronic structure varies considerably. Even under a small electric field [see Fig. 2(c)], a sizable Zeeman-like splitting is observed at the valance bands, whose magnitude at the $\Gamma$ point is comparable to the electrostatic potential difference between adjacent SLs. These states possess different spin textures. On the edge of conduction bands, the Rashba-like splitting can be observed but the splitting is much smaller compared with that of the valance bands. To understand such phenomenon, we calculate the charge density distribution in real space for split states on the conduction and valance bands (see Supplemental Material [39]). We found that the 


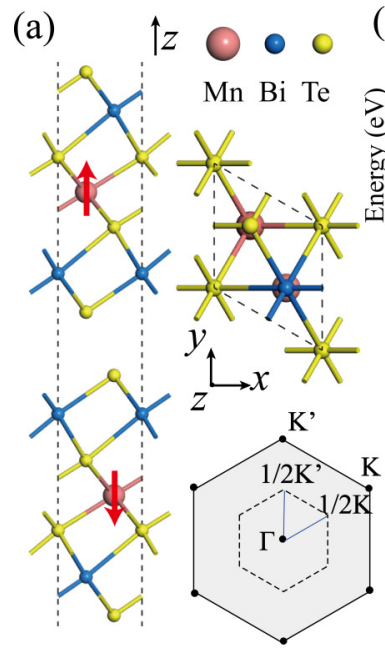

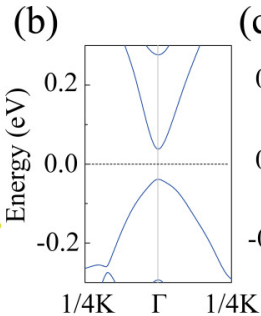

(c)

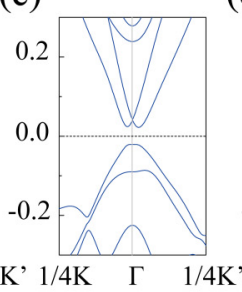

(h)

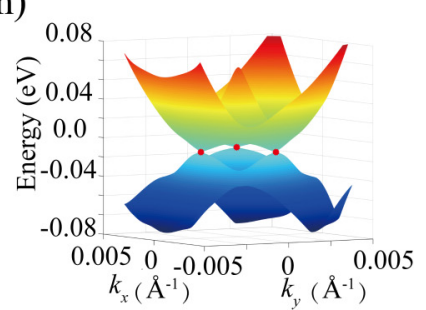

(d)

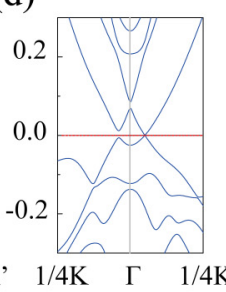

(i) (e)

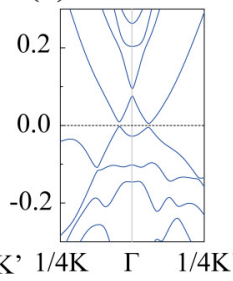

(j) (f)

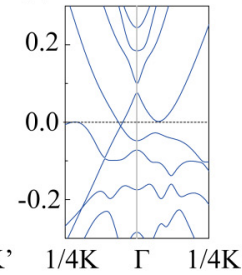

(g)

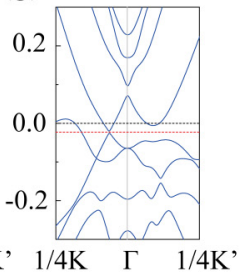

(k)

FIG. 2. (a) Lattice structure of double-SL $\mathrm{MnBi}_{2} \mathrm{Te}_{4}$ thin film and the first BZ. Red arrows mark magnetic orientations on Mn atoms. Band structures of double-SL $\mathrm{MnBi}_{2} \mathrm{Te}_{4}$ thin film under electric fields: (b) $E=0 \mathrm{~V} / \AA$, (c) $E=0.010 \mathrm{~V} / \AA$, (d) $E=0.021 \mathrm{~V} / \AA$, (e) $E=0.023 \mathrm{~V} / \AA$, (f) $E=0.027 \mathrm{~V} / \AA$, and (g) $E=0.029 \mathrm{~V} / \AA$. The Fermi levels marked by the black dashed lines are set to be zero. (h) $2 \mathrm{D}$ electronic structures around the Fermi level and the $\Gamma$ point when electric field is $0.021 \mathrm{~V} / \AA$. Band crossing points are marked as red stars. (i) Schematic of edge states when the double-SL $\mathrm{MnBi}_{2} \mathrm{Te}_{4}$ thin film is a QAH insulator with a Chern number of 3 . The magnetic Mn atoms are indicated by the purple balls with arrows and the black arrow indicates the direction of the electric field. Distributions of the Berry curvature in the first BZ for double-SL $\mathrm{MnBi}_{2} \mathrm{Te}_{4}$ thin film under (j) $E=0.021 \mathrm{~V} / \AA$ and (k) $E=0.029 \mathrm{~V} / \AA$. Corresponding energy levels are marked as red dashed lines in (e) and (g).

valance band edge is mainly contributed by the $p_{z}$ orbital of Te atoms localized on different SLs. They are partner states under $\mathcal{P} \mathcal{T}$ symmetry when the electric field is zero, whose spin polarizations are locked with the SL index. The out-ofplane external field can shift these spin-polarized bands easily, and the energy splitting has the same order of magnitude as the electrostatic potential difference between different SLs. Correspondingly, these bands carry nonzero Berry curvatures. A similar argument has been used to understand the tuning of Berry curvature and valley magnetic moments in bilayer $\mathrm{MoS}_{2}$ [40]. The band edges of conduction bands are contributed by $p$ orbitals of $\mathrm{Bi}$ atoms and Te atoms in the same SL. Thus the out-of-plane electric field will enhance the Rashbalike splitting.

When increasing the electric field, band splittings of valence and conduction bands become larger and larger. We then find topological phase transitions in the double-SL $\mathrm{MnBi}_{2} \mathrm{Te}_{4}$ thin film. The first critical value $E_{c 1}$ is $0.021 \mathrm{~V} / \AA$. Figure 2(d) shows its band structure along high symmetric lines, and the 2D electronic structure for two low-energy bands around the $\Gamma$ point is displayed in Fig. 2(h). The band crossing points are along lines of $\Gamma-\mathrm{K}^{\prime}$. Because the applied electric field is along the $z$ direction, it does not break $\mathcal{C}_{3 z}$ symmetry; then three gapless points are observed in the first BZ. Figure 2(j) shows the momentum-resolved distribution of the Berry curvature $\boldsymbol{\Omega}(\mathbf{k})$ at $E_{c 1}$. Different from the conventional topological phase transition that just hosts singularities of Berry curvature at the crossing points [41], we find a triangle region around the $\Gamma$ point with small gap size, in which all states have large $\boldsymbol{\Omega}(\mathbf{k})$. Beyond $E_{c 1}$, double-SL $\mathrm{MnBi}_{2} \mathrm{Te}_{4}$ becomes an AFM QAH system with a high Chern number of 3, as shown in Fig. 2(i). This result is a proposal to realize the QAH effect with high Chern numbers based on realistic AFM materials. If we further increase the electric field beyond the second critical point $\left(E_{c 2}=0.027 \mathrm{~V} / \AA\right.$ ) , the gap closing process occurs along lines of $\Gamma-\mathrm{K}$. Then the double-SL $\mathrm{MnBi}_{2} \mathrm{Te}_{4}$ becomes a trivial AFM metal. Due to the breaking of $\mathcal{P} \mathcal{T}$ symmetry, this metallic AFM thin film has the nonzero Berry curvature distribution [see Fig. 2(k)], contributing to an intrinsic AH signal. But its value is not quantized anymore. When we flip the direction of the electric field and keep the magnetic structures, the AH signals change sign correspondingly. It is also noted that the band gaps and the critical fields of the system depend on the choice of exchange-correlation functionals. However, the engineering of electronic structures by using the electric field should be robust and independent on the functionals. Besides, we have adopted sufficient thickness of the vacuum layer to make sure the calculations are converged (see details in the Supplemental Material [39]).

In order to confirm the predicted nontrivial band topology of the double-SL $\mathrm{MnBi}_{2} \mathrm{Te}_{4}$ thin film under certain electric fields, we build the 2D tight-binding model with semi-infinite boundary conditions to calculate the edge states, whose effective hopping terms are obtained from ab initio calculations. In Figs. 3(a) and 3(c), we plot the band structure of edge states and $\mathrm{AH}$ conductance as a function of chemical potential in double-SL $\mathrm{MnBi}_{2} \mathrm{Te}_{4}$ thin film under an electric field of $0.023 \mathrm{~V} / \AA$. Inside the $2 \mathrm{D}$ bulk gap, we can observe three chiral edge states clearly. They connect the conduction and valance bands, contributing to the quantized $\mathrm{AH}$ conductance of $\sigma_{x y}=3 e^{2} / h$ [see Fig. 3(c)]. Besides the topologically nontrivial edge states, some other edge states are also found in Fig. 3(a) along the line of $\Gamma-K$. These edge states are topologically trivial without the intrinsic contribution to the $\mathrm{AH}$ conductance.

Figure 3(b) demonstrates the AH signal as a function of chemical potential and external perpendicular electric field that can be well controlled via dual-gate technology. In the 
(a)
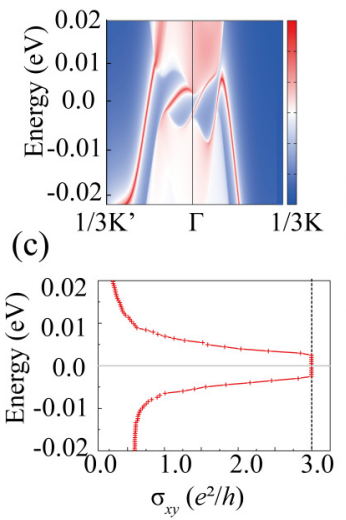

(b)

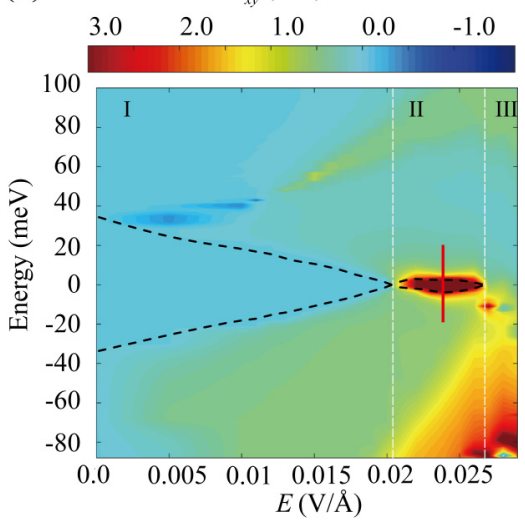

FIG. 3. (a) Edge states in double-SL $\mathrm{MnBi}_{2} \mathrm{Te}_{4}$ thin film under the electric field of $E=0.023 \mathrm{~V} / \AA$. (b) $\mathrm{AH}$ signal as a function of chemical potential and external perpendicular electric fields. The chemical potentials are aligned to Fermi levels. The phase regions I, II, and III represent the trivial semiconducting phase, QAH phase, and trivial AFM metal phase, respectively. The black dashed lines show the energy positions of the conduction and valence band edges in phase regions I and II. (c) AH conductance with varying chemical potential in double-SL $\mathrm{MnBi}_{2} \mathrm{Te}_{4}$ thin film under the electric field of $E=0.023 \mathrm{~V} / \AA$. The chemical potential scale is marked as the red solid line in (b).

trivial semiconducting phase [marked as phase I in Fig. 3(b)], we observe a zero AH signal inside the band gap and the negative value when tuning the Fermi level to the conduction band with minor electron doping. That is mainly contributed by the small energy splitting at the anticrossing point on the conduction band (see details in Supplemental Material [39]). With increasing the electric field, such an anticrossing gap becomes larger and the value of corresponding negative Berry curvature becomes smaller; thus the $\mathrm{AH}$ signal decreases and even changes the sign. By further increasing the electric field, we obtain the quantized AH signal inside the band gap that is guaranteed by the nontrivial band topology of double-SL $\mathrm{MnBi}_{2} \mathrm{Te}_{4}$ thin film [see phase II in Fig. 3(b)]. The topological gap between $E_{c 1}$ and $E_{c 2}$ is $8 \mathrm{meV}$. And this thin film becomes a trivial AFM metal in phase III when the electric field is beyond $E_{c 2}$. Its $\mathrm{AH}$ signal has a finite value when the chemical potential is zero but increases rapidly when we shift down the Fermi level to lower energies (see details in Supplemental Material [39]). The corresponding electronic states are spinpolarized and host large Berry curvature.

The drastic change of AH signal caused by varying chemical potential and electric field offers an opportunity to design AFMS devices via dual-gate technology. Figures 4(a) and 4(b) show the proposed device prototype of the AFM digital circuit (DC) and dynamic random-access memory (DRAM). We use the standard dual gate to simultaneously control the perpendicular electric field and Fermi level to encode the information as the "write-in" process and use the Hall bar to detect the $\mathrm{AH}$ conductance as the "read-out" process. In principle, the AH conductance in "Off" state is zero and its value in "On" state could be as large as $3 e^{2} / h$, so its ideal on/off ratio is infinite. In the realistic experimental conditions with dissipation and at finite temperatures, the double-SL
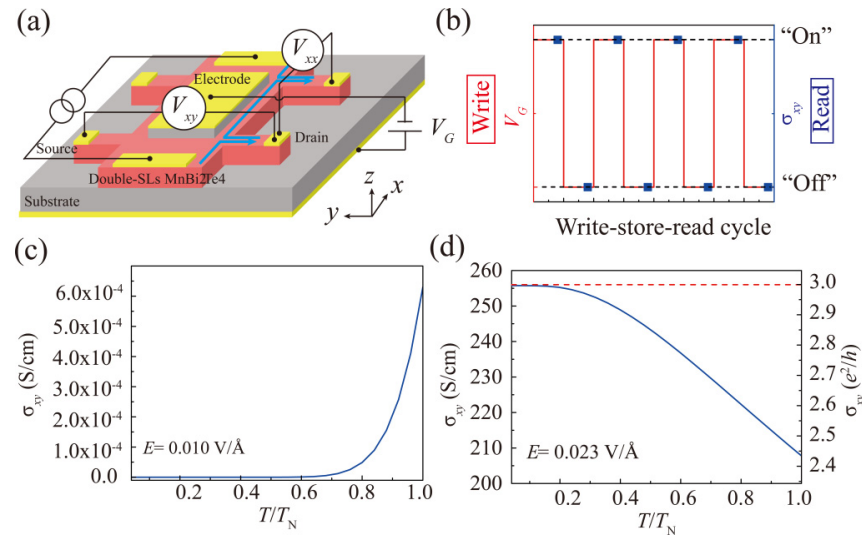

FIG. 4. (a) Top view of schematics for a Hall bar device. The blue arrows indicate the edge current direction. The red areas show the double-SL $\mathrm{MnBi}_{2} \mathrm{Te}_{4}$ thin film. The gray and yellow areas represent the substrate and attached electrodes, respectively. (b) Schematic operation for the AFM memory device. "On" and "Off" states are for double-SL $\mathrm{MnBi}_{2} \mathrm{Te}_{4}$ thin film in the trivial semiconductor phase and QAH phase. (c), (d) AH conductance with varying temperature in double-SL $\mathrm{MnBi}_{2} \mathrm{Te}_{4}$ thin film under the electric field of $E=0.010$ and $0.023 \mathrm{~V} / \AA$. The temperature is normalized to Néel temperature $T_{N}=25 \mathrm{~K}$.

$\mathrm{MnBi}_{2} \mathrm{Te}_{4}$ thin film could be supported on substrates with large dielectric constant, such as $\mathrm{BN}$, silicon, and $\mathrm{SrTiO}_{3}$. The contacting interface might induce an effective electric field that slightly breaks the $\mathcal{P} \mathcal{T}$ symmetry. The $\mathrm{AH}$ conductance in "Off" state thus gains a finite value. In order to simulate such an effect, we put a double-SL $\mathrm{MnBi}_{2} \mathrm{Te}_{4}$ thin film on the $\mathrm{BN}$ substrate and estimate the effective electric-field strength (see details in the Supplemental Material [39]). Then we evaluate its performance at different working temperatures. The calculated results are shown in Figs. 4(c) and 4(d). With increasing the temperature, more states are thermally excited and the on/off ratio becomes smaller, but its value $\left(10^{6}-10^{14}\right)$ is still much larger than the currently realized AFM RAM [6-8,1114]. In our current calculations, we mainly consider the intrinsic Berry-phase-related AH effect. Some other extrinsic mechanisms (e.g., skew scattering) may also contribute to $\mathrm{AH}$ signals [15], but their contributions should be insignificant, because, in contrast to the magnetic alloys, $\mathrm{MnBi}_{2} \mathrm{Te}_{4}$ thin films are cleaved from high-quality single crystals with lower density of impurities and disorders [36-38]. The intrinsic mechanism should dominate the contribution of AH effect.

Beyond the double SL, the discussed physics and proposed spintronic device prototype in this work could be generalized to other $\mathrm{MnBi}_{2} \mathrm{Te}_{4}$ thin films with even SL thickness as long as $\mathcal{P} \mathcal{T}$ symmetry is not intrinsically broken. As the confirmation, we apply the external electric field to a four-SL $\mathrm{MnBi}_{2} \mathrm{Te}_{4}$ thin film that was regarded as an axion insulator [30] (see details in Supplemental Material [39]). We can still observe the spin-polarized band splitting and topological phase transitions driven by the electric field, while its critical values are smaller than those in double-SL $\mathrm{MnBi}_{2} \mathrm{Te}_{4}$, and the topologically nontrivial phase region becomes narrower. To conclude, we believe that the electric field is an efficient method to manipulate the Berry curvature effects in even-SL 
$\mathrm{MnBi}_{2} \mathrm{Te}_{4}$ thin films, resulting in a large change of $\mathrm{AH}$ signal. Thus the even-SL $\mathrm{MnBi}_{2} \mathrm{Te}_{4}$ thin film is a promising material platform to build the low-power AFM memory bit based on the AH signal with electric write-in and read-out. We expect its performance to be stable and robust under the external magnetic field. This work paves the way for using even-SL $\mathrm{MnBi}_{2} \mathrm{Te}_{4}$ thin films, and perhaps AFM topological thin films more generally, in a new generation of electrically switchable AFMS devices.

S.D., J.L., Z.L., Y.X., and W.D. acknowledge financial support from the Basic Science Center Project of the
NSFC (Grant No. 51788104), the Ministry of Science and Technology of China (Grants No. 2016YFA0301001, No. 2018YFA0307100, and No. 2018YFA0305603), the National Natural Science Foundation of China (Grants No. 11674188 and No. 11874035), and the Beijing Advanced Innovation Center for Future Chip (ICFC). A.R. and P.T. acknowledge financial support from the European Research Council (ERC-2015-AdG-694097). P.T. acknowledges funding received from the European Union Horizon 2020 research and innovation program under Marie Sklodowska-Curie Grant Agreement No. 793609. The Flatiron Institute is a division of the Simons Foundation.
[1] V. Baltz, A. Manchon, M. Tsoi, T. Moriyama, T. Ono, and Y. Tserkovnyak, Rev. Mod. Phys. 90, 015005 (2018).

[2] O. Gomonay, T. Jungwirth, and J. Sinova, Phys. Status Solidi RRL 11, 1700022 (2017).

[3] T. Jungwirth, X. Marti, P. Wadley, and J. Wunderlich, Nat. Nanotechnol. 11, 231 (2016).

[4] A. H. MacDonald and M. Tsoi, Philos. Trans. R. Soc., A 369, 3098 (2011).

[5] A. Manchon, J. Železný, I. M. Miron, T. Jungwirth, J. Sinova, A. Thiaville, K. Garello, and P. Gambardella, Rev. Mod. Phys. 91, 035004 (2019).

[6] J. Železný, H. Gao, K. Výborný, J. Zemen, J. Mašek, A. Manchon, J. Wunderlich, J. Sinova, and T. Jungwirth, Phys. Rev. Lett. 113, 157201 (2014).

[7] X. F. Zhou, J. Zhang, F. Li, X. Z. Chen, G. Y. Shi, Y. Z. Tan, Y. D. Gu, M. S. Saleem, H. Q. Wu, F. Pan, and C. Song, Phys. Rev. Appl. 9, 054028 (2018).

[8] X. Marti, I. Fina, C. Frontera, J. Liu, P. Wadley, Q. He, R. J. Paull, J. D. Clarkson, J. Kudrnovský, I. Turek, J. Kuneš, D. Yi, J.-H. Chu, C. T. Nelson, L. You, E. Arenholz, S. Salahuddin, J. Fontcuberta, T. Jungwirth, and R. Ramesh, Nat. Mater. 13, 367 (2014).

[9] X. Z. Chen, R. Zarzuela, J. Zhang, C. Song, X. F. Zhou, G. Y. Shi, F. Li, H. A. Zhou, W. J. Jiang, F. Pan, and Y. Tserkovnyak, Phys. Rev. Lett. 120, 207204 (2018).

[10] M. Wang, W. Cai, D. Zhu, Z. Wang, J. Kan, Z. Zhao, K. Cao, Z. Wang, Y. Zhang, T. Zhang, C. Park, J.-P. Wang, A. Fert, and W. Zhao, Nat. Electron. 1, 582 (2018).

[11] T. Moriyama, W. Zhou, T. Seki, K. Takanashi, and T. Ono, Phys. Rev. Lett. 121, 167202 (2018).

[12] P. Wadley, B. Howells, J. Železný, C. Andrews, V. Hills, R. P. Campion, V. Novák, K. Olejník, F. Maccherozzi, S. S. Dhesi, S. Y. Martin, T. Wagner, J. Wunderlich, F. Freimuth, Y. Mokrousov, J. Kuněs, J. S. Chauhan, M. J. Grzybowski, A. W. Rushforth, K. W. Edmonds, B. L. Gallagher, and T. Jungwirth, Science 351, 587 (2016).

[13] S. Y. Bodnar, L. Šmejkal, I. Turek, T. Jungwirth, O. Gomonay, J. Sinova, A. A. Sapozhnik, H.-J. Elmers, M. Kläui, and M. Jourdan, Nat. Commun. 9, 348 (2018).

[14] K. Olejník, V. Schuler, X. Marti, V. Novák, Z. Kašpar, P. Wadley, R. P. Campion, K. W. Edmonds, B. L. Gallagher, J. Garcés, M. Baumgartner, P. Gambardella, and T. Jungwirth, Nat. Commun. 8, 15434 (2017).
[15] N. Nagaosa, J. Sinova, S. Onoda, A. H. MacDonald, and N. P. Ong, Rev. Mod. Phys. 82, 1539 (2010).

[16] R. Karplus and J. M. Luttinger, Phys. Rev. 95, 1154 (1954).

[17] G. Sundaram and Q. Niu, Phys. Rev. B 59, 14915 (1999).

[18] N. Sivadas, S. Okamoto, and D. Xiao, Phys. Rev. Lett. 117, 267203 (2016).

[19] N. Kiyohara, T. Tomita, and S. Nakatsuji, Phys. Rev. Appl. 5, 064009 (2016).

[20] J. Liu and L. Balents, Phys. Rev. Lett. 119, 087202 (2017).

[21] A. K. Nayak, J. E. Fischer, Y. Sun, B. Yan, J. Karel, A. C. Komarek, C. Shekhar, N. Kumar, W. Schnelle, J. Kübler, C. Felser, and S. S. P. Parkin, Sci. Adv. 2, e1501870 (2016).

[22] S. Nakatsuji, N. Kiyohara, and T. Higo, Nature (London) 527, $212(2015)$

[23] H. Chen, Q. Niu, and A. H. MacDonald, Phys. Rev. Lett. 112 017205 (2014)

[24] Z. Liu, H. Chen, J. Wang, J. Liu, K. Wang, Z. Feng, H. Yan, X. Wang, C. Jiang, J. Coey, and A. H. MacDonald, Nat. Electron. 1, 172 (2018).

[25] Q. L. He, X. Kou, A. J. Grutter, G. Yin, L. Pan, X. Che, Y. Liu, T. Nie, B. Zhang, S. M. Disseler, B. J. Kirby, W. Ratcliff II, Q. Shao, K. Murata, X. Zhu, G. Yu, Y. Fan, M. Montazeri, X. Han, J. A. Borchers, and K. L. Wang, Nat. Mater. 16, 94 (2017).

[26] P. Tang, Q. Zhou, G. Xu, and S.-C. Zhang, Nat. Phys. 12, 1100 (2016).

[27] L. Šmejkal, J. Železný, J. Sinova, and T. Jungwirth, Phys. Rev. Lett. 118, 106402 (2017).

[28] Y. Gong, J. Guo, J. Li, K. Zhu, M. Liao, X. Liu, Q. Zhang, L. Gu, L. Tang, X. Feng, D. Zhang, W. Li, C. Song, L. Wang, P. Yu, X. Chen, Y. Wang, H. Yao, W. Duan, Y. Xu et al., Chin. Phys. Lett. 36, 076801 (2019).

[29] M. Otrokov, I. Klimovskikh, H. Bentmann, D. Estyunin, A. Zeugner, Z. Aliev, S. Gaß, A. Wolter, A. Koroleva, A. Shikin et al., Nature (London) 576, 416 (2019).

[30] D. Zhang, M. Shi, T. Zhu, D. Xing, H. Zhang, and J. Wang, Phys. Rev. Lett. 122, 206401 (2019).

[31] J. Li, Y. Li, S. Du, Z. Wang, B.-L. Gu, S.-C. Zhang, K. He, W. Duan, and Y. Xu, Sci. Adv. 5, eaaw5685 (2019).

[32] M. M. Otrokov, I. P. Rusinov, M. Blanco-Rey, M. Hoffmann, A. Y. Vyazovskaya, S. V. Eremeev, A. Ernst, P. M. Echenique, A. Arnau, and E. V. Chulkov, Phys. Rev. Lett. 122, 107202 (2019). 
[33] A. Zeugner, F. Nietschke, A. U. B. Wolter, S. Gaß, R. C. Vidal, T. R. F. Peixoto, D. Pohl, C. Damm, A. Lubk, R. Hentrich, S. K. Moser, C. Fornari, C. H. Min, S. Schatz, K. Kißner, M. Ünzelmann, M. Kaiser, F. Scaravaggi, B. Rellinghaus, K. Nielsch et al. Chem. Mater. 31, 2795 (2019).

[34] J. Cui, M. Shi, H. Wang, F. Yu, T. Wu, X. Luo, J. Ying, and X. Chen, Phys. Rev. B 99, 155125 (2019).

[35] X.-Y. Dong, S. Kanungo, B. Yan, and C.-X. Liu, Phys. Rev. B 94, 245135 (2016).

[36] Y. Deng, Y. Yu, M. Z. Shi, Z. Guo, Z. Xu, J. Wang, X. H. Chen, and Y. Zhang, Science 367, 895 (2020).

[37] C. Liu, Y. Wang, H. Li, Y. Wu, Y. Li, J. Li, K. He, Y. Xu, J. Zhang, and Y. Wang, Nat. Mater. 19, 522 (2020).

[38] J. Ge, Y. Liu, J. Li, H. Li, T. Luo, Y. Wu, Y. Xu, and J. Wang, arXiv:1907.09947.

[39] See Supplemental Material at http://link.aps.org/supplemental/ 10.1103/PhysRevResearch.2.022025 for methods of calculation; the electronic band structures from HSE06, magnetic properties, and the Berry curvatures with different chemical potentials for double-SL $\mathrm{MnBi}_{2} \mathrm{Te}_{4}$ thin films under an electric field; and the electronic structures for four-SL $\mathrm{MnBi}_{2} \mathrm{Te}_{4}$ thin film with and without electric field, which includes Refs. [42-48].

[40] S. Wu, J. S. Ross, G.-B. Liu, G. Aivazian, A. Jones, Z. Fei, W. Zhu, D. Xiao, W. Yao, D. Cobden, and X. Xu, Nat. Phys. 9, 149 (2013).

[41] S. Murakami, New J. Phys. 9, 356 (2007).

[42] G. Kresse and J. Furthmüller, Comput. Mater. Sci. 6, 15 (1996).

[43] J. P. Perdew, K. Burke, and M. Ernzerhof, Phys. Rev. Lett. 77, 3865 (1996).

[44] S. Grimme, J. Comput. Chem. 27, 1787 (2006).

[45] S. L. Dudarev, G. A. Botton, S. Y. Savrasov, C. J. Humphreys, and A. P. Sutton, Phys. Rev. B 57, 1505 (1998).

[46] J. Heyd, G. E. Scuseria, and M. Ernzerhof, J. Chem. Phys. 118, 8207 (2003).

[47] G. Pizzi, V. Vitale, R. Arita, S. Blgel, F. Freimuth, G. Géranton, M. Gibertini, D. Gresch, C. Johnson, T. Koretsune, J. IbañezAzpiroz, H. Lee, J.-M. Lihm, D. Marchand, A. Marrazzo, Y. Mokrousov, J. I. Mustafa, Y. Nohara, Y. Nomura, L. Paulatto et al., J. Phys.: Condens. Matter 32, 165902 (2020).

[48] Q. Wu, S. Zhang, H.-F. Song, M. Troyer, and A. A. Soluyanov, Comput. Phys. Commun. 224, 405 (2018). 